\title{
A Proposed Solution and Future Direction for Blockchain based Heterogeneous Medicare data in Cloud Environment
}

\author{
Harleen Kaur ${ }^{1}$, M. Afshar Alam ${ }^{1}$, Roshan Jameel ${ }^{1}$, Ashish Kumar Mourya ${ }^{1}$, Victor Chang ${ }^{2}$ \\ 1. Jamia Hamdard University, New Delhi, India \\ 2. IBSS, Xi'an Jiaotong-Liverpool University, Suzhou, China
}

\begin{abstract}
The healthcare data is an important asset and rich source of healthcare intellect. Medical databases, if created properly, will be large, complex, heterogeneous and time varying. The main challenge nowadays is to store and process this data efficiently so that it can benefit the human. Heterogeneity in healthcare sector in the form of medical data is also considered to be one of the biggest challenges for researcher. Sometimes it is referred as large-scale data or big data. Blockchain technology in cloud environment has proved their usability separately. Though, these two platforms can be combined to enhance the exciting applications in healthcare industries at hefty. Blockchain is a highly secured and decentralized networking platform of multiple computers called nodes. It is changing the way the medical information being stored and shared. It makes the work easier, keeps an eye on the security and accuracy of the data and also reduces the cost of maintenance. A Blockchain based platform is proposed that can be used for storing and managing electronic medical records in cloud environment.
\end{abstract}

Keywords: Blockchain technology, Blockchain based cloud, Decentralized Network, Electronic Health Records (EHRs)

\section{Introduction}

Many hospitals are adapting Electronic Health Records (EHRs) that are digital records of the patients, that gives quicker access to the records whenever required. They are responsible to deliver the chances to improve the patient care, to boost the clinical performances and to promote the research in the health sector. The health care data is constantly increasing and in order to handle the healthcare data efficiently, specific technologies are required, like distributed data network, parallel processing, scalable storages, infrastructures, frameworks etc. Since cloud computing is Service Oriented Architecture, it solves these complex issues in virtual environment with minimal cost. The cloud is economical and customizable and only asks to pay for the services and resources that are to be used. It provides infrastructure, platform and software as services. Implementation and migration of Electronic Medical Records (EMR) to cloud based technology and platform such as applications and websites have been considered for accessing and sharing between different health care experts and research labs, enabling more rapid and suitable exchange of data which was not possible earlier [1].

Benefit of using cloud in healthcare system is it gives an archives for storing the medical records and reports. It can provide ways of collaboration for doctors through video calling, healthcare specific mobile applications etc., can help the patients in case of emergencies and can also support rural healthcare. It can also help in analysing the data and help the users to yield good results in spotting the trend, referencing good doctors, medicines or labs, medical research etc. But by using the cloud infrastructure, the users trust the third party i.e., the cloud service provider with their data. Moreover the data in cloud is not encrypted so privacy and security are the main concern of the data owners and cloud service providers because the health care data that is being stored on the cloud carries sensitive 
information and the attackers are constantly trying the new ways to enter the system. So, in order to deal with these issues, in medical databases usage of blockchain in the cloud is proposed.

Using blockchain technology for health information can transform the healthcare industry. As the healthcare industry is moving toward patient centric models, all the components are being framed to benefit the patient. The data in Medical databases, if created properly, will be large, complex, heterogeneous and time varying. However using blockchain for storing and managing the Electronic Health Records will be efficient and secure. The blockchain on cloud environment for handling healthcare data will provide high quality service at a relatively low cost.

The proposed model is the implementation of blockchain network on cloud environment and it is going to cover every aspect of the health industry from doctor, patient, pharmacies to insurance provider, government etc. The proposed model can have multiple applications in the medical industry as it can be used for patient record management in which all the medical history of the patient can be saved like the symptoms, tests conducted, possible disease and the medications. Other useful application of the model could be, managing the insurance claim policies by the insurance provider and the patient. It can also be used in medical research, interested researcher can easily look up into the saved data for analysing any disease with respect to age, gender or geographical conditions etc.

The transaction forms in the blockchain are created using programming language, and can be duplicated, to avoid this, contract-transactions can be used for the verification purposes [2, 3, 4]. In contract based transactions, a script is used that contains multiple signatures for better security. Another type of issue that might occur in blockchain architecture is a bug. Although blockchain are supposed to be the safest and most reliable networking platforms but the occurrence of a bug is unpredictable, as happened with the bitcoin a bug named CVE-2010-5139 occurred and lasted for about 8 hours, this means that a bug is also a threat to the blockchain architecture [5]. Therefore while implementing the blockchain technology, one should take into consideration these issues and challenges that have been encountered earlier to avoid complications in future.

This paper gives an outline on the implementation and applications of using blockchain technology in cloud for storing and managing the healthcare data. A literature review on the technologies is given in the next section, which is followed by the concept of blockchain and cloud computing with respect to medical system. A Blockchain model for blockcloud is proposed and the concept, architecture, and its internal working is mentioned in detail. In next section how blockchain is suitable for handling heterogeneous data and the need of its interoperability is also discussed which is followed by the discussion and conclusion.

\section{Related Works}

In recent years, healthcare sector has changed dramatically due to boom in clinical research, and to provide better facilities and data sharing among different domains. In this section, different healthcare research and medical drifts pertaining to healthcare data, patient information through cloud services providers and access rule with prominence on the improving blockchain technology are summarized.

The concept of blockchain was first introduced in a white paper report, which pointed out the challenges of Crypto-currencies and their solution in the form of blockchain technology proceeding to the completion of transaction [6]. Blockchain technology has been successfully applied for managing the access control on a platform called Enigma where the different users can store the data and perform computations while the data remains private [7]. Another such system called ChainAnchor was proposed that allows the anonymous entities to perform transactions on the system after their identities 
being verified using Enhanced Privacy ID [8]. The blockchain concept is also used in collaboration with IoT for secure accessing of the smart contracts [9].

Blockchain technology has gained considerable deliberation in recent years with increased curiosity in several diverse fields like Fiscal and Medical. Medical data hold personal and sensitive information that may be attractive to cyber criminals. The curiosity and impetus has now extended to medical industry. [43] focuses on the different Blockchian technology application in health care. While in the early stages of design and development, various organizations have projected novel technologies that have the latent to maximize medical data like heterogeneous transparency and operating capabilities. Though the security, cost effectiveness and scalability of new blockchain technology will entail more research proceeding to the big data invention deployments.

Health care industries are data concentrated domain where a huge amount of data is generated, stored, scattered and accessed daily. Blockchain applications for client, patient medical data portability, interoperability, care and delivery management and supervision can provide the answer to many challenges that are being faced by healthcare industries. So, there has been tremendous concentration in utilizing blockchain technology in the provision of secure medical data management [44].

The Enhanced trusted model for patient's medical data sharing proposed by [10] has highly secured data encryption and decryption techniques. This model needs permission of Blockchain network to share patient's information among medical practitioner. The proposed model encrypted the patient information before sending it through blockchain network. Security features are modified in the model due to restricted counterfeit. Ophthalmology based apps for decision support system has been proposed recently by [11]. This mobile system based apps has played a vital role in mining different specialist's decision on a particular issues rose by someone, who hasn't enough knowledge in the field of ophthalmology for diagnose the disease.

Medical professional have currently witnessed the problem of heterogeneity in unstructured data generated by different heterogeneous sources. [12] proposed the networking solution for medical professionals that use semantic information automatically for merging different domain data and extract useful information for diagnoses. MedRec: Architecture based on distributed network for storing and accessing Electronic health records through smart contracts proposed by [13]. The main principle of architecture based blockchain technology. MedRec has highly secured authentication process and share patient information among the experts. It has also highlighted and proposed solution for mining big data.

A rural health care information system based on cloud on a high-level which provides minimal cost facilities to the people of rural areas proposed by [14]. They have stored personal information in cloud based system accessed by medical practitioner and researchers for better medical facilities and diagnosis of remote diseases. Patients have special privileges to access their health records and prescriptions any time for quick treatment. This system is cheaper in comparison to other and eliminated the time and other processes needed to enrol in a hospital.

To reduce human generated error in patient data, sensor base equipments and cloud computing technology can play significant role in error free data collection and diagnosis.[15] proposed automated system for health care sector by using a cloud based architecture for capturing information through sensor's. This data can be accessed by domain experts and can be distributed to medical practitioners and staff for diagnosis. Its principle is also based on cost reduction mechanism and provides better facilities to patients. It also reduces labour cost and is highly secured because of its two tier security. 
Data integrity is a challenging problem for researchers in computing area. Even, it is more complicated in cloud computing where different platform user farm out more data. A solution that has been inspired by European project SUNFISH [16]. The proposed solution has highlighted the preliminary of Blockchain based storage and cloud computing. They have focused on high-level security protocols for accessing and authentication of data integrity.

Cloud computing is a new technique, that provide different services by minimizing cost and infrastructure. Its transformation in healthcare industries can be beneficial on high level. Nkosi et. al., [17] proposed a cloud based protocol management system that provides sensing device signal processing and high security as a service to different mobile devices. System has reassured another mobile device from executing heavier multimedia and security algorithms in delivering mobile health services. These improve the deployment of the ubiquitous mobile device for shared services and support health service delivery to marginalized rural areas.

Currently the data deletion from cloud computing is a considerable topic of interest for researcher's because the server from cloud side may not erase the complete records sincerely for fiscal incentives. [41] proposed a new data erasing scheme using blockchain technology that is more transparent. In this technique, the owner of data can authenticate the deletion consequences no matter how nastily the cloud server performs. Suppose the owner of A data sets on cloud server (cS) don't completely rely on each other. The blockchain technology assures the owner about the deletion request on the $\mathrm{cS}$ cloud server to erase the particular record (R). Then $\mathrm{cS}$ validates the request from authentication process and verify them. Now after erasing the particular record, the $\mathrm{cS}$ cloud server and another timestamp server sent the data deletion proof to the owner of the data.

Cloud computing and mobile computing are used by [42] with blockchain system for sharing information in a distributed scheme. A new prototype system has been proposed focusing on blockchain component. Performance investigation has also been conducted for this blockchain component. No centralized servers are used to process and store shared data. Due to mobile computing platform, all the communication and mining process perform through virtual terminals as an alternative of mobile terminals. The blockchain based client server computing has ensured data integrity and traceability.

MeDShare is a healthcare data sharing via cloud computing using blockcahin technology. It has been proposed by [18]. They have used smart contract and authentication permission on data access through different platform. The proposed system analyzed the performance of system by comparing the presently edge solution of data sharing through different cloud service companies. MeDShare has ability to securely achieve and maintain data provenance and auditing at the same time as sharing healthcare data between another cloud structure.

ProvChain is a cloud based data provenance architecture proposed by [19] for enhancing availability and Privacy issues. This technology is totally decentralized and use cloud computing for fiddle proof access by using blockchain technology.

\section{Blockchain Technology for Medical Data}

Big data and cloud computing paradigm in medical sector enables the revolution from theoretical driven research to semantic data research by gathering large scale of heterogeneous data in medical domain. Different health related information in medical sector is the main reference for medical people diagnosing and caring sick patients. With the tremendous explosion in information technology, off-line medical data have progressively been transformed into online mode. Large volumes of data is being generated by the organizations including medical sector, finances, business evaluation, customer's 
activities tracking [20-21, 47], website information etc. Furthermore with the arrival of social networking applications and medical sector related websites, the individuals are also generating the data in huge amount on daily basis.

The term Big Data refers to the management, analysis, processing and visualization of this data [22-23]. Knowledge Discovery in Data is a technique that is currently being used for analyzing the patterns in the data for better decision-making, and for finding out the relation between the non-related data attributes Data Mining is used [24, 25]. Big Data Analytics constitutes the techniques like KDD, Data Mining; Machine Learning based predictive models, Quantitative Analysis etc. to get the benefit out of the data available $[45,46]$. Big Data in medicine can benefit in predicting the disease and provide better diagnosis and treatment to the patients. The big data integrates information about multiple features of diseases and try to learn and evolve a better solution to the human being.

Big data in medical sector generated from different resources, such as electronic health records, patient data warehouses, healthcare measurements, scanning and imaging. The medical data and health records have to be assessed and construed in an appropriate way to benefit medical sector. But medical practitioners need highly innovative tools and applications to track, outline, and offer fast response for individual users. Appropriate data mining will also assist health services and healthcare policies. Big Data analytics requires high computing and storage for handling medical data. In [26] author discussed on various data types in big data make it complex and needs more computation capabilities to analyze such a large data set. And cloud could be used as an effective platform to deal with such problem. The cloud computing can have various meanings for different peoples, like a user can use the cloud for storing the data and accessing it from anywhere and anytime, while for researcher it could be a place from where the data can be collected for some analysis or research, whereas for organizations, it could be a safer and cheaper option to store the data and find out some relevant information from it to improve the business. The infrastructures offered by the clouds are IaaS (Infrastructure as a Service), PaaS (Platform as a Service) and SaaS (Software as a Service). The cloud gives pay as you go facility to the clients and allows the processing of data efficiently. While the big data is responsible for storing and processing huge amount of data cloud provides an environment that is scalable and reliable [27]. And in [28] the author states that cloud computing services are best suitable for performing large-scale complex computational tasks.

Medical sector may be intelligent to acquire their initial steps in the direction of implementing cloud based big data elucidation by defining patients who will use the clinical information and investigation offered by large scale data. By converting offline data into online and efficiently using big data, medical organizations and healthcare sector lying from single medical practitioner and multinational companies to large healthcare insurance networks stand to grasp noteworthy benefits. Prospective advantages contain early diagnosis of diseases as they can be used more simply and successfully; treating specific entity, detecting medical insurance fraud faster and easily [29].

In [30] a series of six steps is provided to deploy the big data analytics into the cloud environment. It considers the business use cases, requirements of the stakeholders, the accountability, privacy and security of the organization's data. It gives a cloud-based platform to the organization to deploy it with the data it generates on day-to-day basis. A paper [31] is published and discussed on the compatibility of cloud computing and big data and address how these two emerging technologies complement each other. However there are numerous uses and applications of big data in cloud infrastructure, but the data privacy and security are the biggest concerns. As cloud service providers are the third parties and the data of organizations is very sensitive it would be a concern to give access of this data to anybody. Also there are some security breaches in cloud and the attackers are always finding out the ways to get 
into the data $[39,40]$. So, in order to have a secure architecture for data storage and transmission, concept of blockchain in the clouds is proposed.

Lack of privacy and security of the confidential data makes the blockchain technology a good choice for healthcare data. Along with the privacy, blockchain would give access control (security) and huge distributed data storage for heterogeneous data. And for storing the data that is not required very frequently off-chain repository could be used. The use of blockchain will offer multiple advantages to the users, having a single source for data storage and access with high security and privacy will be useful for the users as well as the researchers.

\section{Blockchain based Platform for Heterogeneous Medical Data}

The big data of medical sector processing abilities of cloud computing technology make easier the investigation of the entire variable at once is a noteworthy enabler of the new field of biotechnology [32] a holistic loom which helps to imagine the 'omics' as a fascinating network, generating a hypothesis shift- permit us to roam from theoretical- driven to theoretical generating research

Cloud computing has appeared as a theory for storing and processing the data and services on the internet. It has attracted the organizations as it allows the enterprises from the medical fields, to start from a small scale and then grow as per their requirements. It only asks to pay for the resources that are being used. It is often called on demand service as it has a pool of resources available at very affordable rates. The cloud can be public, private and hybrid. It is totally up to the client, what type of cloud they need for their business. The cloud does not require any physical space or the user knowledge; this makes the health care management easier. But the only concern with cloud is with the data privacy, as with cloud, the organizations will have to trust the cloud service providers with their data. And for that, the usage of blockchain in the cloud environment is proposed.

Blockchain technology is a highly secured and decentralized networking platform of multiple computers called nodes. It can change the way the information is being stored and shared. It makes the work easier, keeps an eye on the security and accuracy of the data and also reduces the cost of maintenance. The blockchain architecture is distributed that makes the data available all the time. The data on blockchain is immutable, that is it cannot be altered and that makes the data fraud resistant. And the blockchain also provides data provenance that verifies the integrity of the data.

All the transactions and data are stored in the blocks after applying encryption. The Blockchain technology has its own protocols that contain the log information about client, timestamp, cryptographical information. The digital signature makes authentication process more secure. The protocol is broadcasted over the network. It could be visible to all the connected nodes but accessible by those for which it has been generated. The blockchain based cloud system is supposed to take full charge of the data transfer, storage or processing. And when the data is on the blockchain, anyone who has the access to that blockchain will know what is going on with the data.

Both cloud computing and blockchain techniques have their own advantages and disadvantages. It is proposed in the paper to integrate them both, to get the best of both the worlds. The model is proposed for the healthcare data. The data will be stored in the cloud therefore it will be accessible to anyone, anywhere and also it will be on the blockchain network, that would make the data secure. It would be like a PaaS (Platform as a Service) where there will be a blockchain based platform for the healcare industry to store, manage and process the data. 


\subsection{Cloud for Medical Data}

The proposed work integrates the medical data into the blockchain based cloud infrastructure as shown in Figure 1. The blockchain technology and cloud computing have proved their usability separately, the proposed framework integrates both the technologies and provides an architecture for real time medical data storage, transfer, sharing and processing of healthcare data that is secured, traceable and affordable.

The heterogeneous medical data that is supposed to be processed will be stored on the blockchain in a distributed environment. And every transaction-taking place will be stored on the interconnected blocks. And since these blocks are decentralized no central entity will own the data, everybody on the network will be considered as the owner. And every transaction will be notified to all nodes on the system. And this data is encrypted before storing using cryptography that saves it from any damage.

Internet has played a vital role in peer-to peer networking and it is an essential backbone of the cloud computing. Networking security needs more attention both from internal as well as external attacks, for this a well defined planning to design blockchain based cloud architecture is required. 


\subsection{Architecture Concept}

The proposed model has three main components Domain Experts including Doctors, Lab Technicians, Pharmacists, Drug Manufacturer etc., Health Insurance Provider and Patient.

All the components will be accessing and/ or generating the data on the cloud using the blockchain technology as shown in Figure 1. The messages will be used to notify if there is any change in the data or there is any transmission. In figure 2 gives a conceptual view of the proposed system.

In the proposed architecture, the transactions will be occurring on the files that are fragmented and stored on various nodes across the whole network after encryption. This ensures the privacy of the data. And also its distributed and decentralized nature makes it resistant to the errors that might occur during the storage or transmission of data.

\subsection{Working of the proposed system}

The methodology and workflow of the proposed system is depicted in figure 3 . The user will send a request to do a transaction. The request will be sent to the proposed blockchain based cloud architecture and it will verify the user's identity using the cryptographic credentials. After the request and user is verified, the system will process the request; this request could be of storing, processing, transferring or retrieving the medical data from the network. When the verified request is granted, a new block is added to the existing blockchain that contains the information of this transaction and the new state of data. The user is provided with what he/ she asked for and the transaction completes.

\section{Internal Workflow of Blockchain based platform}

Blockchain has ledger in which the transaction medicine data is stored and this ledger is updated with every new transaction, hence maintaining the integrity of the data. It is a P2P based architecture and eliminates the need of third party for any transaction. This type of system is easier to implement and scale. The internal workflow of blockchain network like how the blocks are connected to another and how a transaction takes place is briefly described in this section.

\subsection{Blocks Connection}

The nodes of blockchain consist of a header along with the body as shown in Figure 4. The body will contain the actual data of the node while the header will have the hash values of the previous node. This is how the nodes will be linked in the blockchain. The data is distributed on the entire network and an indexing technique is used to go through the nodes [33]. And since the data in one node is related to the previous node, it makes it impossible to alter the data in the network. This ensures the integrity of the data. First node of the blockchain does not have any previous node for the reference and is supposed to be generated on its own and then all other nodes will be added afterwards. Also the concept of cryptography is included in the architecture that guarantees the ownership of the data, as the owner will hold the private key and other nodes will be provided with the public key [34]. This makes the owner provenance possible. 
Using the blockchain for health data in cloud will ensure the privacy of the data, as there is no third party involved, and the data will be distributed after applying cryptography over the nodes that will make it more secure. The transactions are all recorded and hence make it more traceable. Because of all these strengths, the blockchain can be effectively used in healthcare.

\subsection{Blockchain Transaction}

Blochchain technology allows the transactions without any central controlling identity. And the transactions are secured with cryptology. And all the details of the transactions are distributed throughout the network that makes it traceable and verifiable. The fundamental element of blockchain architecture is the address of the nodes. It tells from where a particular node or block belongs.

The main component of the transaction procedure is linking these nodes. The figure 5 shows how the information flows during transactions. Basically the data flows between the nodes based on the addresses. As shown in the previous section, that a block contains the data and the address of the next node as output. The transaction is secured using a series of signatures called e-stamp. And the data is transferred from one node to the next along with the hash value of the previous node along with the estamp. The receiver will confirm the owner of the data by looking at the e-stamp. And the identity will be verified using this stamp and public key of previous node.

\section{A Blockchain model for Handling Heterogeneous Medical Data}

The migration of healthcare records to cloud based technology has ease the sharing of patient information among medical sector and researchers, enabling quicker and more opportune exchange in a way before not possible. A peer-to-peer blockchain technology that contains medical data records, documents or EEG signals would have data handling inferences and healthcare throughput constraints. These types of healthcare data have been proven its difficulty in managing through off-chain as well as on-chain, due to its heterogeneity in nature. Heterogeneous data means any data with heterogeneity in nature. Heterogeneity defines high variability of different formats and nature. Healthcare sector takes it as an assortment of data types that contains qualms and incompleteness of diverse degrees that mixed an extra component to the innovative heterogeneity. Several machine learning techniques and methods of Data mining haven't handled it well. Many solutions have been introduced to reveal the handling of heterogeneous data through visualizing information and mapping between structured data schemas. The problem of heterogeneity in unstructured data applications creates myth to discover knowledge discovery database that perform efficient heterogeneous data mining to handle various types of data sources. Heterogeneous (imbalanced) data define as the objects depicted by various features of different types, forms and architectures.

The medical data may have different data types for example, the details of a patient, like patient id, bill no., age will stored in numerics and some of the symptoms like body temperature, blood pressure, blood sugar level etc. will be stored in numerical form as well. While details like gender, disease, will be written in non-numerical form, symptoms like pain intensity will be in ordinal form, also there will be data like X-Ray, Ultrasound, MRI reports in the form of images, and also documents like medical laboratory report, along with some data in the form of signals like ECG, Mobile Spectrum etc. [35]. By combining these datatypes we can have some useful information.

Storing patient information in the form of health record, straight on the block-chain certify that the data is stored through the highly secured transaction. The block-chain technology does not store the heterogeneous data types directly to the blockchain such as X-rays or ECG signal data. It is rather stored off-chain. To store it off-chain, the heterogeneous data would require pointer to a new address 
location. When a user be it a patient, or a healthcare service provider creates a patient medical record or prescription (such as personal information ECG, MRI) an e-Stamp would be generated to verify user authenticity of prescription as shown in figure 6 . The heterogeneous data would be converted through encryption techniques and sent to the cloud storage.

Blockchain Technology presents many benefits to healthcare sector for managing heterogeneous data. Researchers of healthcare industries need broad and heterogeneous data types for understanding the disease, genetic information and enlargement of drug. The shared information made available by blockchain technology would distribute a broad assorted dataset by patient and users from various racial, socio-economic platforms and geographical areas. Blockchain would guarantee constant accessibility to real-time information.

Real time accessibility to patient information would advance clinical care harmonization and improve patient care in emergency cases. Real time patient information must also permit public health care industries and medical researchers to quickly detect, segregate for environment circumstance that blow public health.

\section{Blockchain Interoperability}

The blockchain technology and distributed ledgers has been the talk of the town in context of cryptocurrency and it was said to be the future of internet as it will work as a trust layer and everything will be lying on it. Because of this reason the usage of blockchain is accelerating in almost every possible direction of real world and real time applications and healthcare is no exception. But to make it last in efficient manner, interoperability of blockchain is required. Because in future the people would like to have other platforms linked with the blockchain, from where they can move their assets to blockchain, or move their assets from blockchains to these platforms. True interoperability can be achieved if the blockchain based platforms starts interacting with other traditional platforms or integrate them or replace them completely.

The multi-chain is a topic of great interest but to make them reality is a difficult process. For example, if we want to get a bitcoin in exchange of money, then we needs a tool for this, we cannot actually convert the money into bitcoin. Or another example, we want to buy some cryptocurrency then we have to make wallet identity to interact with the currency provider, this identity can be generated from various available extensions, and for every wallet we need to have individual ids, and cannot login to any wallet without having the id or with id generated on different wallet. Hence, different identity wallets are required for different extensions. The interoperability tries to bridge this gap and will allow the user to have only one wallet identity for all the extensions.

Amber Baldet, blockchain program lead, J.P Morgan chase said in Money2020, that interoperability barriers could be threefold; interoperability with the existing platforms, interoperability in same types of blockchains and interoperability in different types of blockchains.

\section{Discussion}

Currently the healthcare data is stored electronically, and the systems are centralised on a small scale. And if we consider rural places, not everybody is familiar with the concept of storing and accessing the data electronically [36]. Therefore, moving the whole data electronically and then into a distributed decentralized network would increase the interoperability but also will be a very time consuming process. And also the exact cost of the system is not known. But collecting the huge amount of data and making a distributed system will require resources and management. And after the implementation it 
would require maintenance for the proper functioning. Thus, the whole implementation will cost a fortune to the government.

The concept of blockchain in healthcare sounds so amazing but it has not been successfully implemented by any country. And since there is no proven use case, [37] nobody wants to take risk. But in order to make blockchain successful multiple funds provider, healthcare leaders and government will have to work together for the transformation of healthcare industry, because once it would be done. It is so going to benefit the end users.

Blockchain is going to cover every aspect of the health industry from doctor, patient, pharmacies to insurance provider, government etc. Therefore, the data amount on the blockchain is going to be huge. There will be lots of transactions per second and the system might get slower [38]. Also the blockchain maintains a log of all the activities occurred so far, so there is data generated with every transaction and along with that the data is stored in every block of the blockchain, so that there is no chance of losing any data but it creates so much redundant data. Hence, to make it work efficiently the technology would require constant upgradation.

\section{Conclusion}

Healthcare data or medical record has been proven its importance for patient, because it is a valuable asset, according to their point of view. The data in healthcare is mostly the personal details of the users and hence shall not be shared with third parties as it is not safe and might be misused. Unfortunately, healthcare data or clinical information are scattered among various medical repositories. Sharing and Accessing Healthcare records are crucially important for intelligence and advanced medical services. Blockchain technology has been demonstrated in e-commerce that trusted, auditable transaction is possible in peer-to-peer networking accomplished by Hash history ledger. In this study, we have proposed a model for healthcare data in blockchain based architecture on cloud computing environment. The paper gives an outline about the framework, its internal working and protocols for handling heterogeneous healthcare data. In future we will work on enterprise data warehouse issues through blockchain technology.

\section{Acknowledgements}

This research work is catalyzed and supported by National Council for Science and Technology Communications (NCSTC), Department of Science and Technology (DST), Ministry of Science and Technology (Govt. of India) for support and motivation [grant recipient: Dr. Harleen Kaur and grant No. 5753/ IFD/ 2015-16]. The authors gratefully acknowledge financial support from the Ministry of Science and Technology (Govt. of India), India.

\section{Compliance with Ethical Standards.}

This research work is catalyzed and supported by National Council for Science and Technology Communications (NCSTC), Department of Science and Technology (DST), Ministry of Science and Technology (Govt. of India) for support and motivation [grant recipient: Dr. Harleen Kaur].

\section{Conflict of Interest. $\quad$ None}




\section{References}

[1] Longo, D.L.; Drazen, J.M., Data Sharing, N. Engl .J. Med. 2016, 374, 276-277.

[2] Bradbury, D. The problem with Bitcoin. Comput. Fraud Secur. 2013, 11, 5-8.

[3] Park, Jin Ho and Jong Hyuk Park. "Blockchain Security in Cloud Computing: Use Cases, Challenges, and Solutions.” Symmetry 9 (2017): 164.

[4] Paul, G.; Sarkar, P.; Mukherjee, S. Towards a more democratic mining in bitcoins. In Proceedings of the International Conference on Information Systems Security, Hyderabad, India, 16-20 December 2014; Springer International Publishing: Cham, Switzerland, 2014.

[5] Eyal, I.; Emin, G.S. Majority is not enough: Bitcoin mining is vulnerable. In Proceedings of the International Conference on Financial Cryptography and Data Security, Christ Church, Barbados, 3-7 March 2014; Springer: Berlin/Heidelberg, Germany, 2014.

[6] Satoshi, N. (2008), Bitcoin: A Peer to Peer Electronic cash system, [online]. Available : http://bitcoin.org/bitcoin.pdf

[7] Zyskind, G.; Nathan, O.; Pentland, A. Enigma: Decentralized Computation Platform with Guaranteed privacy. arXiv 2015.

[8] Hardjono, T.; Pentland, A.S. Verifiable Anonymous identities and Access Control in Permissioned Blockchains. manuscript in preparation (2016).

[9] Ouaddah, A.; Elkalam, A.A.; Ouahman, A.A. Towards a novel privacy-preserving access control model based on blockchain technology in IoT. Europe and MENA Cooperation Advances in Information and Communication Technologies pp.523-533, 2017.

[10] Kim, K.J. and Hong, S.P., A Trusted Sharing Model for Patient Records based on Permissioned Blockchain, . Journal of Internet Computing and Services (JICS), 6, pp.75-84, 2017.

[11] De la Torre-Díez, Isabel, Borja Martínez-Pérez, Miguel López-Coronado, Javier Rodríguez Díaz, and Miguel Maldonado López. "Decision support systems and applications in ophthalmology: literature and commercial review focused on mobile apps." Journal of medical systems 39, no. 1 (2015): 174.

[12] Kerkri, E.M., Quantin, C., Allaert, F.A., Cottin, Y., Charve, P., Jouanot, F. and Yétongnon, K., 2001. An approach for integrating heterogeneous information sources in a medical data warehouse. Journal of Medical Systems, 25(3), pp.167-176.

[13 ] Azaria A, Ekblaw A, Vieira T, Lippman A., Medrec: Using blockchain for medical data access and permission management. InOpen and Big Data (OBD), International Conference on 2016 Aug. 22 (pp. 25-30). IEEE.

[14] Padhy, R. P., Patra, M. R., \& Satapathy, S. C. (2012). Design and implementation of a cloud based rural healthcare information system model. Univers J Appl Comput Sci Technol, 2(1), 149-157.

[15] Rolim, C. O., Koch, F. L., Westphall, C. B., Werner, J., Fracalossi, A., \& Salvador, G. S. (2010, February). A cloud computing solution for patient's data collection in health care institutions. In eHealth, Telemedicine, and Social Medicine, 2010. ETELEMED'10. Second International Conference on (pp. 95-99). IEEE.

[16] Gaetani, E., Aniello, L., Baldoni, R., Lombardi, F., Margheri, A. and Sassone, V., 2017. Blockchain-based database to ensure data integrity in cloud computing environments. ITA-SEC.CEUR-WS.org.

[17] Nkosi MT, Mekuria F. Cloud computing for enhanced mobile health applications. Proceedings of the 2010 IEEE 2nd International Conference on Cloud Computing Technology and Science (CloudCom); The 2nd IEEE International Conference on Cloud Computing Technology and Science; Nov 30- Dec 3, 2010; Indianapolis, USA. New York, NY: IEEE; 2010. 
[18] Sharma, Pradip Kumar, Seo Yeon Moon, and Jong Hyuk Park. "Block-VN: A distributed blockchain based vehicular network architecture in smart City." Journal of Information Processing Systems 13, no. 1 (2017): 84.

[19] Liang, Xueping, Sachin Shetty, Deepak Tosh, Charles Kamhoua, Kevin Kwiat, and Laurent Njilla. "Provchain: A blockchain-based data provenance architecture in cloud environment with enhanced privacy and availability." In Proceedings of the 17th IEEE/ACM International Symposium on Cluster, Cloud and Grid Computing, pp. 468-477. IEEE Press, 2017.

[20]Attention, shoppers: Store is tracking your cell, New York Times. http://www.nytimes.com/2013/07/15/business/attention-shopper-storesare-tracking-your-cell.html.

[21] Unlocking Game-Changing Wireless Capabilities: Cisco and SITA help Copenhagen Airport Develop New Services for Transforming the Passenger Experience, Customer case study, CISCO (2012).

[22] Bell, G., Hey, T. and Szalay, A., Beyond the data deluge. Science, 323(5919), pp.1297-1298.,2009.

[23] Franks, B., Taming the big data tidal wave: Finding opportunities in huge data streams with advanced analytics (Vol. 49). John Wiley \& Sons, 2012

[24] Fayyad, U., Piatetsky-Shapiro, G. and Smyth, P., The KDD process for extracting useful knowledge from volumes of data. Communications of the ACM, 39(11), pp.27-34.,1996.

[25] Witten, I.H., Frank, E., Hall, M.A. and Pal, C.J., Data Mining: Practical machine learning tools and techniques. Morgan Kaufmann, 2016.

[26] Talia, D., Clouds for scalable big data analytics. Computer, 46(5), pp.98-101., 2013.

[27] Hashem, I.A.T., Yaqoob, I., Anuar, N.B., Mokhtar, S., Gani, A. and Khan, S.U., The rise of "big data" on cloud computing: Review and open research issues. Information Systems, 47, pp.98-115., 2015.

[28] Huan, L. Big data drives cloud adoption in enterprise, IEEE Internet Comput. 17 (2013) 68-71.

[29] Raghupathi, Wullianallur, and Viju Raghupathi. "Big data analytics in healthcare: promise and potential." Health information science and systems 2, no. 1 (2014): 3.

[30] James Kobielus, Deploying Big Data to the Cloud: Roadmap for Success http://www.cloud-council.org/webinars/CSCCWebinar-Big-Data-in-the-Cloud-Roadmap-for-Success-July-31-2014-Kobielus.pdf.

[31] James Kobielus, I., \& Bob Marcus, E. S. (2014). Deploying Big Data Analytics Applications to the Cloud: Roadmap for Success. Cloud Standards Customer Council.

[32] O’Driscoll, Aisling, Jurate Daugelaite, and Roy D. Sleator. "'Big data', Hadoop and cloud computing in genomics." Journal of biomedical informatics 46, no. 5 (2013): 774-781.

[33] Ziegeldorf, J.H.; Matzutt, R.; Henze, M.; Grossmann, F.; Wehrle, K. Secure and anonymous decentralized Bitcoin mixing. Future Gener. Comput. Syst. 2016.

[34] Kachouri, R., Djemal, K. and Maaref, H. Multi-model classification method in heterogeneous image databases. Pattern Recognition, 43(12), pp.4077-4088, 2010.

[35] Natoli, C.; Gramoli, V. The blockchain anomaly. In Proceedings of the 2016 IEEE 15th International Symposium on Network Computing and Applications (NCA), Cambridge, MA, USA, 2016.

[36] http://news.childrensmercy.org/6-challenges-to-healthcare-blockchain-adoption.

[37] http://www.freedassociates.com/wp-content/uploads/2017/08/Blockchain_White_Paper.pdf .

[38] https://www.healthit.gov/sites/default/files/4-37-hhs_blockchain_challenge_deloitte_consulting_llp.pdf.

[39]Wu, Z. Y., Lee, Y. C., Lai, F., Lee, H. C., and Chung, Y., A secure authentication scheme for telecare medicine information systems. J. Med. Syst. 36(3):1529-1535, 2012. 
[40] Chen, C. L., and Yang, T. T., A secure medical data exchange protocol based on cloud environment. J. Med. Syst. 2014. doi: 10.1007/s10916-014-0112-3.

[41] Yang, C., Chen, X. and Xiang, Y., Blockchain-based publicly verifiable data deletion scheme for cloud storage. Journal of Network and Computer Applications, 103, pp.185-193,2018

[42] Ho, Yik Him, Ziv Cheng, Peter Man Fai Ho, and Henry CB Chan. "Mobile Intercloud System with Blockchain." In Proceedings of the International MultiConference of Engineers and Computer Scientists, vol. 1. 2018.

[43] Angraal, S., Krumholz, H.M. and Schulz, W.L., Blockchain technology: applications in health care. Circulation: Cardiovascular Quality and Outcomes, 10(9), p.e 003800, 2017

[44] Esposito, C., De Santis, A., Tortora, G., Chang, H. and Choo, K.K.R., Blockchain: A Panacea for Healthcare Cloud-Based Data Security and Privacy?. IEEE Cloud Computing, vol. 5(1), pp.31-37, 2018

[45] Chauhan, R., Kaur, H.: Predictive analytics and data mining: a framework for optimizing decisions with R tool. In: Tripathy, B., Acharjya, D. (eds.) Advances in Secure Computing, Internet Services, and Applications (pp. 73-88). Information Science Reference, Hershey.

[46] Kaur, H., Chauhan, R., Alam, M.: Spatial Clustering Algorithm using R-tree. Journal of Computing 3(2), 85-90 (2011)

[47] Kaur H, Chauhan R, Zafar Ahmed (2012) Role of data mining in establishing strategic policies for the efficient management of healthcare system - a case study from Washington DC area using retrospective discharge data. BMC J Health Serv Res 12(Suppl 1) : P12 